\section{Tied to the mast}

\section{By Lauren Martz, Staff Writer}

Long-lasting, nontoxic adjuvants that enhance the often weak or insufficient immune response from nasal vaccines have been hard to find. ${ }^{1}$ A paper in Nature Medicine now suggests that small molecule mast cell activators might function as intranasal vaccine adjuvants to enhance the antigen-specific immune response. ${ }^{2}$ But given the role of mast cell activation in allergy and asthma, some companies question whether such activators can be used as adjuvants without causing inflammatory or anaphylactic reactions. ${ }^{3}$

The paper, by Duke University Medical Center researchers, built on previous work in the same lab that showed that tumor necrosis factor (TNF) released from mast cells is necessary for lymphocyte recruitment to draining lymph nodes, a process essential for adaptive host defense. $^{4}$

In the new study, the researchers show that activated mast cells can also initiate an antibody response. Subcutaneous or intranasal administration of the mast cell activator compound $48 / 80$ (C48/80) in combination with Bacillus anthracis protective antigen (PA) led to significantly higher $\mathrm{PA}$-specific serum IgG levels in wild-type mice compared with those in mast cell-deficient mice $(p<0.05)$.

Also, immunization with $\mathrm{C} 48 / 80$, a polyamine that can induce mast cell degranulation, ${ }^{5}$ plus the B5R poxvirus protein protected significantly more mice from nasal challenge with vaccinia virus than did immunization using B5R alone $(p=0.0325)$.

Previous work by the Duke group and other researchers suggests that mast cells ramp up antigen-specific immune responses by releasing inflammatory mediators including TNF and histamine, which enhance recruitment of lymphocytes and dendritic cells (DCs) to the draining lymph nodes. ${ }^{6}$ The increased DC migration is thought to be due to higher expression of DC surface receptor CC chemokine receptor 7 (CCR7), which is required for DCs to migrate to lymph nodes. ${ }^{7}$

Alfonso Martin-Fontecha, lecturer in immunology at King's College London and Guy's and St. Thomas' Hospital, told SciBX the published data suggest that upregulation of CCR7 might be responsible for the increased DC migration, but cautioned that the results do not rule out other potential mechanisms of DC recruitment.

Although the Duke researchers suggest that C48/80 causes CCR7dependent DC recruitment to the lymph nodes, Martin-Fontecha said that mast cell activation can also promote monocyte recruitment from the blood to inflamed skin.

Stephen Galli, professor of pathology and of microbiology and immunology at Stanford University School of Medicine, also pointed out that the mast cell-deficient Kit mice used as comparators in the study may have deficiencies in other immune effector cells. Thus, the failure of these knockout mice to respond to mast cell activators may have resulted from defects in other cell types besides mast cells, including neutrophils, he said.

Soman Abraham, corresponding author on the paper, told SciBX that to fully test the hypothesis that mast cells are responsible for the immune response observed in mice, "future approaches to replenish mast cell-deficient mice with mast cells might be to isolate mast cell progenitor cells from bone marrow and inject these into mast cell-deficient mice."

Abraham is professor and director of graduate studies in pathology at the medical center.

Whatever the mechanism by which mast cell activators work, Galli said that "from a clinical perspective, if this novel vaccination approach can be shown to be effective and safe, it may be of considerable value even if the method works because of effects on cells other than or in addition to mast cells."

\section{Targeted activation}

Company representatives and academics polled by SciBX had mixed views on whether mast cell activators could have commercial applications in the adjuvant space. One group sees the activators as potentially protective nasal vaccine adjuvants that directly target immune cells. Other researchers are wary of potential safety issues.

"Unlike intramuscular immunization strategies, immunization along the nasal route is needle-free and an effective way to generate not only systemic immunity but also mucosal immunity," said Abraham. "Many pathogens enter the body through mucosal routes. Therefore, generation of prophylactic nasal vaccines that generate protection along these regions is

highly desirable."

George Kemble, VP of R\&D and general manager of the vaccines division at MedImmune Inc., a subsidiary of AstraZeneca plc, said that in many cases, nasal adjuvants are essential because healthy people can "clear particles, such as subunit vaccines, as they enter the nasal passage before they have a chance to stimulate the cells of the innate immune system."

MedImmune markets FluMist, a live attenuated intranasal influenza vaccine. It is the only intranasal vaccine approved in the U.S.

Kemble noted that FluMist "elicits a large enough cellular response on its own" and does not require an adjuvant. However, he told SciBX that "as we turn our attention to other vaccines, both intranasal and non," mast cell activators "look to be quite interesting." 
The vaccine space needs "a new class of adjuvants that are targeted" to cells directly involved in immune stimulation, according to Sudhir Agrawal, CEO and CSO of Idera Pharmaceuticals Inc. Aluminumbased adjuvants are not targeted to such cells and are therefore associated with unwanted side effects, he added.

Idera is developing toll-like receptor (TLR) agonists as targeted adjuvants. In 2006, Idera granted Merck \& Co. Inc. an exclusive worldwide license to develop and commercialize TLR agonists for use in the pharma company's vaccines for treating and preventing cancer, infectious diseases and Alzheimer's disease (AD).

"TLR9 is specifically presented on DCs and B cells, making these receptors ideal targets for immune cell-specific activation," said Agrawal.

Urban Ramstedt, senior director of immunology at Elusys Therapeutics Inc., added that using mast cell activators to trigger immunostimulatory cytokine release at the site of infection could reduce the chances of causing inflammatory adverse events compared with using systemic delivery of immunostimulatory cytokines.

Elusys' Anthim has completed a Phase I safety study in humans and has shown efficacy in nonhuman primates. The affinity-enhanced $\mathrm{mAb}$ that targets anthrax toxin PA has Fast Track designation from the FDA and is being developed under the agency's animal efficacy rule, which allows marketing approval to be based on efficacy data from animal models and safety data in humans.

\section{Potential for inflammation}

Other company representatives and academics were less sanguine about the prospects of using mast cell activators as adjuvants. The key concern is the potential for inflammation.

"Because mast cells are involved in allergic reactions," said Larry Smith, VP of vaccine research at Vical Inc., "there is an underlying concern about the ability to drive appropriate immune response."

The company's Vaxfectin, a cationic lipid-based adjuvant, is in Phase I testing for influenza.

Indeed, Abraham noted that his team saw "that mice had a transient nasal irritation at higher doses of $\mathrm{C} 48 / 80$. However, these effects resolved on their own. Moreover, C48/80 did not induce antigen-specific IgE anti- bodies or increase total IgE levels, which would otherwise indicate that the activators were inducing allergic hypersensitivity.

Going forward, Abraham told SciBX that studies of mast cell activators "will be conducted in primates, with special emphasis on the potential for adverse effects, including inflammation at the site of administration, [and] systemic inflammatory effects, including anaphylactic response and alterations in blood pressure or respiration."

Smith added, "The difficulty in dealing with novel adjuvant approaches such as mast cell activators is to develop compounds that provide sufficient stimulation of the adaptive response without triggering excessive reactogenicity. Assuming safe and effective doses can be established in nonhuman primates, a required next step would be preclinical safety and toxicology studies eventually paving the way to dose-escalation studies in humans." It can be difficult to develop compounds that do not cross the line between sufficient stimulation of immune cells to initiate the adaptive immune response and stimulation to the point of unwanted inflammation. ${ }^{8}$

\section{REFERENCES}

1. Ogra, P. et al. Clin. Microbiol. Rev. 14, 430-445 (2001)

2. McLachlan, J. et al. Nat. Med.; published online April 20, 2008; doi:10.1038/nm1757

Contact: Soman N. Abraham, Duke University Medical Center, Durham, N.C. e-mail: soman.abraham@duke.edu

3. Marshall, J.S. \& Bienenstock, J. Curr. Opin. Immunol. 6, 854-859 (1994)

4. McLachlan, J. et al. Nat. Immunol. 4, 1199-1205 (2003)

5. Norrby, K. Virchow's Arch. B Cell Pathol. Incl. Mol. Pathol. 38, 57-65 (1981)

6. Suto, H. et al. J. Immunol. 176, 4102-4112 (2006)

7. Ohl, L. et al. Immunity 21, 279-288 (2004)

8. Pashine, A. et al. Nat. Med. 11, S63-S68 (2005)

COMPANIES AND INSTITUTIONS MENTIONED

AstraZeneca plc (LSE:AZN; NYSE:AZN), London, U.K. Duke University Medical Center, Durham, N.C.

Elusys Therapeutics Inc., Pine Brook, N.J.

Guy's and St. Thomas' Hospital, London, U.K. Idera Pharmaceuticals Inc. (NASDAQ:IDRA), Cambridge, Mass. King's College London, London, U.K. Medlmmune Inc., Gaithersburg, Md.

Merck \& Co. Inc. (NYSE:MRK), Whitehouse Station, N.J. Stanford University School of Medicine, Stanford, Calif. Vical Inc. (NASDAQ:VICL), San Diego, Calif. 